Rel at i onshi ps bet ween muscl e power out put using the stretch-shorteni ng cycle and eccent ri c maxi mum st rength

\begin{tabular}{|c|c|}
\hline 著者 & M yaguchi Kazuy oshi, Demur a Shi ni chi \\
\hline $\begin{array}{l}\text { jour nal or } \\
\text { publ i cat } i \text { on } t i t l e\end{array}$ & Journal of Strength and Condi ti oni ng Research \\
\hline vol une & 22 \\
\hline nunber & 6 \\
\hline page $r$ ange & 1735- 1741 \\
\hline year & 2008 11- 01 \\
\hline URL & ht t p: //hdl . handl e. net /2297/19131 \\
\hline
\end{tabular}




\title{
Relationships Between Muscle Power Output Using the Stretch-Shortening CyCle AND Eccentric Maximum Strength
}

\author{
Kazuyoshi Miyaguchi $^{1}$ and Shinichi Demura ${ }^{2}$ \\ ${ }^{1}$ Ishikawa Prefectural University, Nonoichimachi, Japan; and ${ }^{2}$ Faculty of Education, Kanazawa University, Kanazazwa, Japan
}

\begin{abstract}
Miyaguchi, $\mathrm{K}$ and Demura, S. Relationships between muscle power output using the stretch-shortening cycle and eccentric maximum strength. J Strength Cond Res 22(6): 1735-1741, 2008-This study aimed to examine the relationships between muscle power output using the stretch-shortening cycle (SSC) and eccentric maximum strength under elbow flexion. Eighteen young adult males pulled up a constant light load $(2 \mathrm{~kg})$ by ballistic elbow flexion under the following two preliminary conditions: 1) the static relaxed muscle state (SR condition), and 2) using the SSC with countermovement (SSC condition). Muscle power was determined from the product of the pulling velocity and the load mass by a power measurement instrument that adopted the weight-loading method. We assumed the pulling velocity to be the subject's muscle power parameters as a matter of convenience, because we used a constant load. The following two parameters were selected in reference to a previous study: 1) peak velocity $\left(\mathrm{m} \cdot \mathrm{s}^{-1}\right)$ (peak power) and 2) 0.1 -second velocity during concentric contraction $\left(\mathrm{m} \cdot \mathrm{s}^{-1}\right)$ (initial power). Eccentric maximum strength by elbow flexion was measured by a handheld dynamometer.

Initial power produced in the SSC condition was significantly larger than that in the SR condition. Eccentric maximum strength showed a significant and high correlation $(r=0.70)$ with peak power in the SSC condition but not in the SR condition. Eccentric maximum strength showed insignificant correlations with initial power in both conditions. In conclusion, it was suggested that eccentric maximum strength is associated with peak power in the SSC condition, but the contribution of the eccentric maximum strength to the SSC potentiation (initial power) may be low.
\end{abstract}

KEY WoRDS rotary encoder, handheld dynamometer, manual muscle testing, countermovement

Address correspondence to Kazuyoshi Miyaguchi, kazu1060@lapis. plala.or.jp.

22(6)/1735-1741

Journal of Strength and Conditioning Research

(c) 2008 National Strength and Conditioning Association

\section{INTRODUCTION}

$\mathrm{M}$ uscle action styles in physical exercise are roughly classified into three types: isometric, concentric, and eccentric muscle actions. In dynamic exercise, eccentric muscle action mostly precedes concentric muscle action. Such an exercise is called a stretch-shortening cycle (SSC) $(22,23)$. Concentric contraction using an SSC produces greater power output in a shorter period of time than a simple concentric contraction by itself $(22,30,39)$.

The use of an SSC with a countermovement is observed in various sports activities and is needed to more effectively perform the main motion itself. The agonist muscle is expanded before the main motion, and this is accompanied by a considerable physical burden. For instance, Fukashiro (13) has reported that a force of five times the weight of an object is generated at the time of a countermovement when pulling up the object using an SSC under elbow flexion. To bear this large load, eccentric strength, functioning as breaking strength, may largely contribute to the power output used in the SSC, rather than isometric or concentric strength. Hence, we hypothesized that muscle power output using an SSC would show significant and high correlations with eccentric maximum strength.

Until now, studies on the measurement, evaluation, and reinforcement of muscle function have been mainly performed using isometric and concentric contractions. Recently, it has been easy to measure eccentric strength through the use of an isokinetic dynamometer. However, it has also been pointed out that the movements measured using this device differ from those actually used in sports settings, which are mainly isotonic muscle contraction movements. This is because the exertion force is measured under specific conditions with controlled acceleration $(17,28)$. Moreover, because the device is very expensive and large, it is very difficult to use it in general sports settings (40). Therefore, we aimed to easily and simply measure eccentric strength by using a handheld dynamometer (HHD), which is widely used in medical institutions.

This study aimed to examine the relationships between muscle power output using SSC and eccentric maximum strength under elbow flexion.

VOLUME 22 | NUMBER 6 | NOVEMBER 2008 1735 


\section{Methods}

\section{Experimental Approach to the Problem}

Until now, studies on SSC have been mainly performed using jumping $(3,6,7,38)$. However, this movement is a multijoint, whole-body movement, and performances are considerably affected by technical factors (e.g., forward and upward arm swings). Furthermore, quantification of muscle power is difficult, because body weight is used as the load (17).

On the other hand, SSC is used frequently in many sports that require the use of the upper limbs, such as baseball, tennis, and golf. Stretch-shortening cycle exercise is indispensable for athletes who require upper-body power. However, the muscle power of the upper limbs has not been extensively studied in comparison with that of the lower limbs. Hence, when evaluating muscle function in relation to sports performance, we have to consider the measurement and assessment of muscle function corresponding to SSC in the upper limbs as well.

Hence, elbow flexion was selected in this study as a motion that is not affected by body weight, that can isolate the muscle groups related to the movement because it is a single-joint movement, and that can use SSC with countermovement easily.

As for power output properties, the jump with multijoint movement differs greatly from the elbow flexion with singlejoint movement; however, the mechanism of SSC in the individual joints is equal. Until now, the mechanism of SSC also has been examined under elbow flexion $(13,35)$. By clarifying the relationship between SSC and strength under elbow flexion, useful information may be obtained that can be applied to future training regimes.

\section{Subjects}

The subjects consisted of 18 young adult males with regular exercise experience (mean age $19.5 \pm 0.9$ years, height $1.73 \pm$ $0.05 \mathrm{~m}$, body mass $64.8 \pm 7.2 \mathrm{~kg}$, and athletic career $4.6 \pm$ 2.5 years). They were selected from the following sports backgrounds: baseball, badminton, soccer, tennis, volleyball, track and field, and so on. Hence, the experience with SSC movement may be different between individuals on the basis of sport specificity. In addition, the subjects did not participate in a periodic training program.

All subjects were judged to be right-handed by Oldfield's (31) handedness inventory. The power test was performed with the dominant arm because of the tendency toward dominant hand use in throwing or hitting. Informed consent was obtained from all subjects after a full explanation of the experimental project and its procedures. This study was approved by the human rights committee of Kanazawa University.

\section{Procedures}

Experimental Device and Muscle Power Measurement. Muscle power was measured using a muscle power measurement device that adopted the weight-loading method developed by Ikemoto et al. (20) (Yagami, Japan) (Figure 1). This device consists of a rotary encoder attached to a fixed pulley and a recording device. The rotary encoder (SUNX, ORE38-1200) can measure the rotational angle with a sampling frequency of $100 \mathrm{~Hz}$ via an analog-to-digital interface. The rotational angle was converted to the pulling velocity of the wire rope with the load in the recording device. The muscle power was drawn from the product of the pulling velocity and the load mass on the basis of Newton's second law of motion.

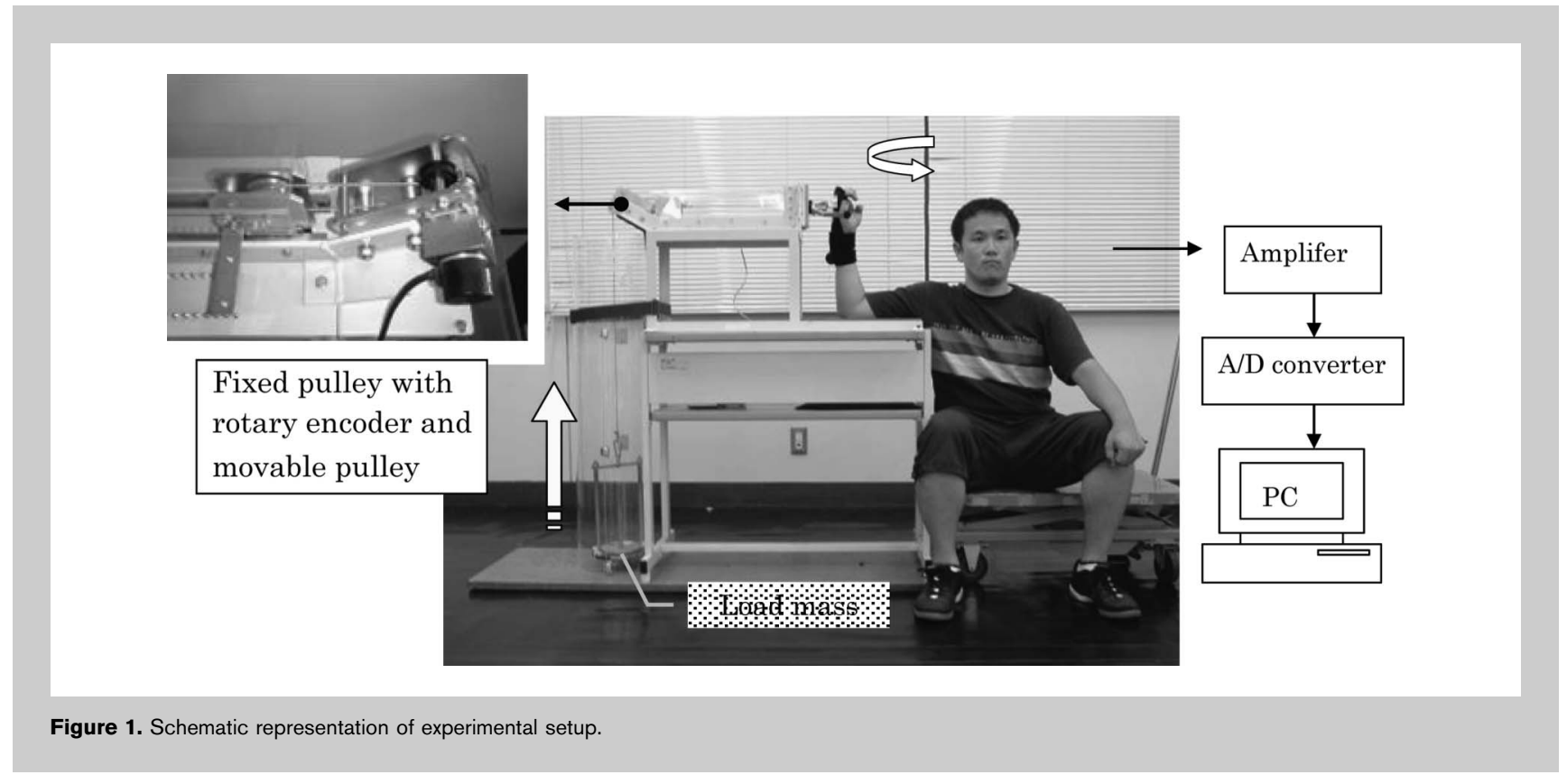

1736

Journal of Strength and Conditioning Research"

Copyright $($ National Strength and Conditioning Association. Unauthorized reproduction of this article is prohibited. 
Each subject sat sideways in an adjustable ergometric chair and put his right arm on a table. Each subject then put his axilla on the edge of the table with supination of the forearm. A bowling protector was worn to restrict the movement of the wrist. Each subject touched his palm to the handle and explosively pulled the handle from the later-stated two preliminary conditions by elbow flexion as quickly as possible in the opposite direction of a wire rope connected to a load mass.

The range of motion of the elbow flexion was from 80 to $120^{\circ}$ (full-extension angle was $0^{\circ}$ ), and the starting position angle was $80^{\circ}$. To account for systematic error, the rotary encoder and load cell were calibrated before each measurement.

Experimental Conditions. To examine the relationship between muscle power output using SSC and eccentric maximum strength, the static relaxed arm muscle state (SR condition) without using SSC was selected as a comparison condition.

1. SR condition: Each subject pulled the handle by only concentric contraction as quickly as possible from a static relaxed arm muscle state while keeping an $80^{\circ}$ elbow joint angle.

2. SSC condition: Each subject pulled the handle with the same load using a voluntary countermovement according to the subject's own rhythm and timing within the range of $80-120^{\circ}$. Because the subject's best SSC performance could not be demonstrated when the restriction of the countermovement was severe, the frequencies of the countermovement were assumed to be arbitrary. The starting angle of the concentric contraction on elbow flexion was determined by a beeping sound from a device at a position of $80^{\circ}$, and several rehearsals were performed by the subject.

Because the subjects have experienced SSC movements of their upper limbs in each sport, they were able to learn the rhythm and timing of the countermovement of this experimental exercise within two or three pretraining sessions. It was assumed that the subjects were exerting maximum power, with the understanding that this was the purpose of the study.

The power test was performed twice for the abovestated two conditions, and the higher value was used in the analysis data. Test-retest reliability at peak power was high (SR: intraclass correlation $[\mathrm{ICC}]=0.85$; $\mathrm{SSC}: \mathrm{ICC}=0.78)$; therefore, it was speculated that each subject was able to exert maximum power consistently.

In the case of using SSC with an upper extremity, it was reported that the effect of prestretching is very large when performing the movement, similar to a flexible spring (36). Hence, when using countermovement, the lightest-intensity load that can recognize weight without producing muscle tone is desirable as the load setting. Thus, in this study we used a constant load of $2 \mathrm{~kg}$ that was less than $10 \%$ of each subject's isometric maximum strength as measured by HHD.

\section{Measurement of Eccentric and Isometric Maximum Strengths}

Eccentric strength is often measured with an isokinetic dynamometer. However, in the case of isokinetic muscle action, it was pointed out that the inhibitory effect of the nervous system is strong and that measured values of eccentric maximal strength become low (15). Hence, the eccentric maximal strength of elbow flexion was measured by an HHD ( $\mu$ Tas F-1, ANIMA), which is used by many medical institutions because of its convenience. In addition, isometric strength was measured as a comparison condition to examine the characteristics of eccentric strength.

Handheld dynamometers can be used to precisely document muscle force in actual units. This HHD is compact and lightweight, and the reliability of measured values is high (2). Eccentric strength was measured in a dorsal position by referring to the manual muscle test (11). The tester stood by the side of the bed, where the subject lay, and grasped the forearm of the subject while the subject maintained a constant posture $\left(90^{\circ}\right.$ elbow joint angle) (Figure 2). The subject was instructed to try to keep the forearm in that position with maximum strength. The tester increased the force through the HHD that was attached to the hand to force the elbow of

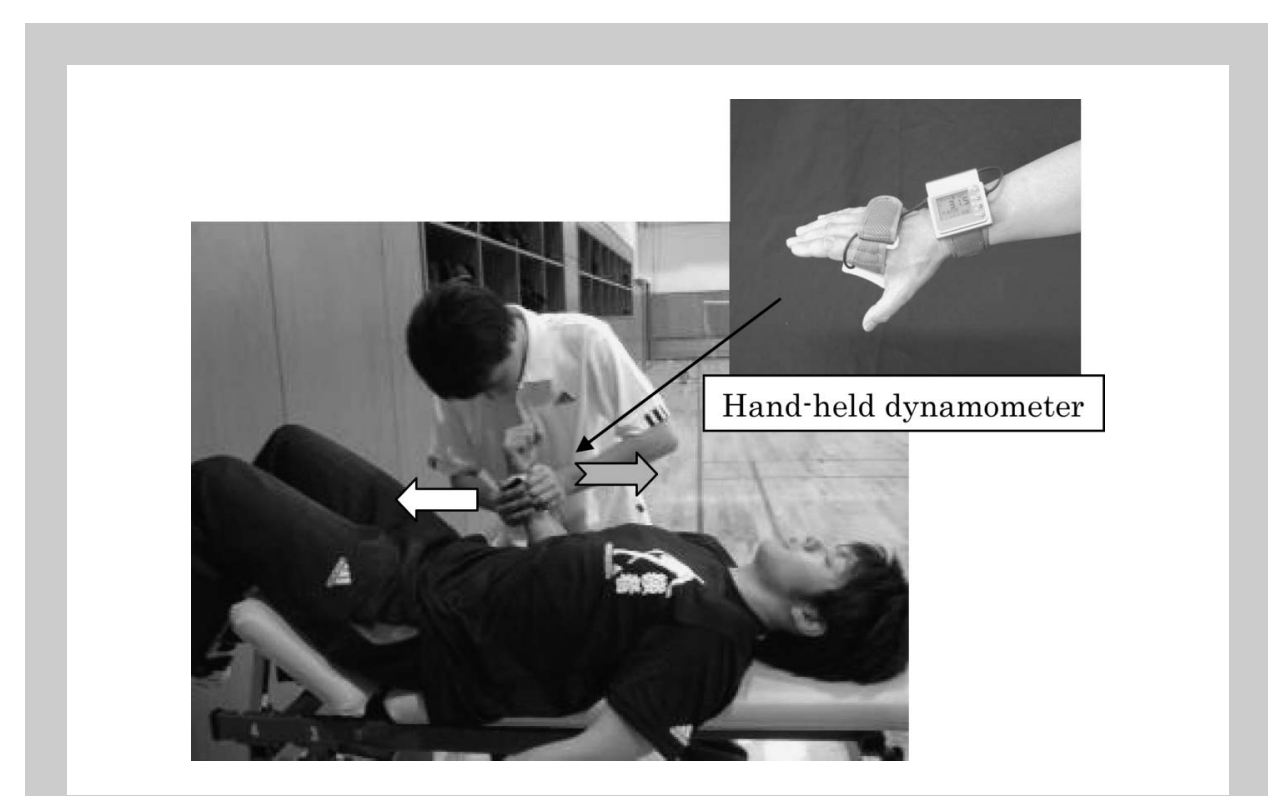

Figure 2. Measurement of eccentric maximum strength. 


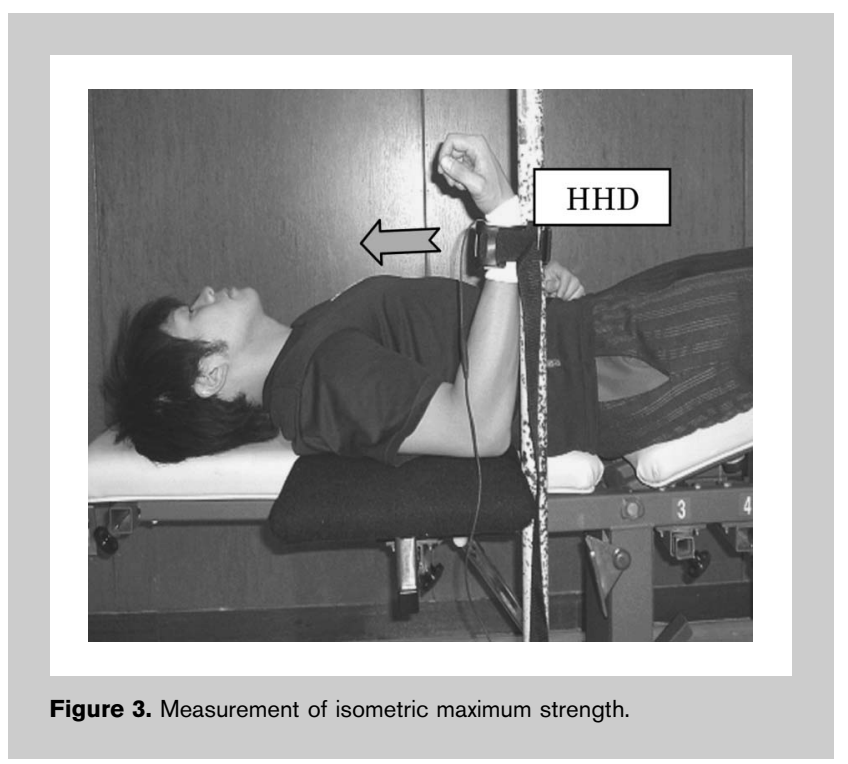

the subject to extend (breaking the posture), and the instantaneous pressure to the HHD when the subject could no longer maintain the same posture was measured.

For measurement of isometric maximum strength, the HHD was fixed to a belt that connected the wrist to a perpendicular post while the elbow joint was maintained at a $90^{\circ}$ angle in the dorsal position (Figure 3). We measured load pressure to the HHD that was exerted when the subject pushed on the fixed HHD by isometric maximum contraction. Eccentric maximum strength was measured first, and after 1 week isometric maximum strength was measured. Both strength tests were performed twice, and the higher value was used in the analysis data.

\section{Evaluation Parameters}

Power is calculated as the product of load weight $\times$ pulling velocity. In this study, we assumed the pulling velocity to be the subject's muscle power parameter as a matter of convenience, because we used a constant load.

The following two parameters were selected in reference to a previous study (29): 1) peak velocity $\left(\mathrm{m} \cdot \mathrm{s}^{-1}\right)$, and 2) 0.1 -second velocity during concentric contraction $\left(\mathrm{m} \cdot \mathrm{s}^{-1}\right)$. It was supposed that the former is peak power and the latter is initial power. In addition, Figure 4 shows the typical time-series velocity curves, muscle contraction types, and evaluation parameters observed in both conditions.

\section{Statistical Analyses}

The reliability of measurement values by HHD was examined by ICC. The paired $t$-test was used to reveal mean differences between isometric and eccentric maximum strengths and between two conditions for muscle power parameters. The relationships between the muscle power parameters of both conditions and eccentric maximum strengths were examined using the Pearson correlation coefficient. The criterion level for significance was set at $p \leq 0.05$.

\section{Results}

The ICC between the two trials of eccentric maximum strength values measured by HHD was high $(r=0.87)$, and that of isometric maximum strength was very high $(r=0.92)$ as well. Table 1 shows the measurement results of eccentric and isometric maximum strengths by HHD. Eccentric maximum strength $(29.7 \pm 4.3 \mathrm{~kg})$ showed a significant and higher value (about 17\%) than isometric maximum strength $(25.6 \pm 4.6 \mathrm{~kg})$ and significant and high correlations $(r=0.76)$ with isometric maximum strength. The ratio of isometric maximum strength to eccentric maximum strength (ISO/ECC, expressed as a percentage) ranged from 97 to $153 \%$.

Table 2 shows the parameters examined in the SR and SSC conditions, the test results of mean differences, and the correlations between each parameter and eccentric maximum strength. The SSC condition showed significantly higher values than the SR condition in 0.1-second velocity but not in peak velocity. As for the relationships between eccentric maximum strength and each parameter, peak power showed a significant and high correlation $(r=0.70)$ in the SSC condition but not in the SR condition. On the other hand, initial power showed insignificant correlations in both conditions.

1738 Journal of Strength and Conditioning Research 
TABLE 1. Measurement results by hand-held dyanometer.

\begin{tabular}{lccc}
\hline & Isometric strength $(\mathrm{kg})$ & Eccentric strength $(\mathrm{kg})$ & Ecc / Iso $\times 100$ \\
\hline Mean & $25.6^{*}$ & $29.7^{*}$ & 117.3 \\
$S D$ & 4.6 & 4.3 & 14.5 \\
Range & $17.3-33.4$ & $20.2-33.6$ & $96.8-153.4$ \\
\hline \multicolumn{2}{c}{${ }^{*} p<0.01}$. & &
\end{tabular}

result is also supported by reports such as those of Walshe et al. (37) or Miyaguchi and Demura (29) where SSC potentiation was found to be marked in the initial power output (0.1-second initial power). As for the upper limbs, very dexterous movement is possible, because they are liberated from antigravity mechanisms. Therefore, the mechanism of power exertion

\section{Discussion}

The reliability of eccentric strength as measured by HHD was high. Hence, HHD may be used in general sports settings. In addition, after measurement of eccentric strength, all subjects reported delayed-onset muscle soreness that was specific for eccentric movement $(8,21)$. At the time of measurement of isometric strength, muscle soreness was not reported. Therefore, it was inferred that enough eccentric muscle activity was caused by the test to measure eccentric maximal strength using the HHD.

Muscle power exertions that use eccentric muscle actions, where muscle and tendon are extended forcibly, can exert larger power outputs than those exerted by concentric or isometric muscle actions (34). Eccentric strength showed a higher value (about 17\%) compared with isometric strength in this study. In addition, eccentric maximum strength showed significant and high correlations $(r=0.76)$ with isometric maximum strength. This suggests that eccentric strength may be reinforced by isometric training that is aimed at the reinforcement of conventional isometric muscle strength. However, the ratio of eccentric maximum strength to isometric maximum strength was $97-153 \%$. Therefore, the above ratio may be considerably different between individuals who differ in training form or competition characteristics.

The potentiation by SSC was confirmed only in the 0.1 -second velocity compared with the SR condition. This using SSC in the upper limbs may be different from the lower limbs (antigravity muscle), in which tension is continuously exerted to maintain one's standing posture. Hence, it was inferred that there were insignificant differences between the SR and SSC conditions in peak power (peak velocity). This suggests that the muscle power properties of the upper limb cannot be properly evaluated with only peak power, as jump performance can.

Hence, we paid great attention to the relationships between 0.1 -second velocity and eccentric maximum strength. We observed that eccentric maximum strength showed insignificant correlations with 0.1 -second velocity in both conditions. From the above, it was suggested that the contribution of the eccentric maximum strength to the SSC potentiation (initial power) may be low.

Aura and Komi (4), Komi and Bosco (24), and Colliander and Tesch (9) have reported that females showed higher values than males in the ability to use elastic energy in eccentric muscle action. Actually, it has been reported that females are superior to males in the ratio of eccentric strength to concentric strength (18). However, even if we considered the physique differences of males and females, females are generally inferior to males in sportive activities (i.e., abilities of throwing and jump) to which SSC strongly contributes. Additionally, although the decrease in eccentric strength with aging is small in comparison with concentric strength (32), the ability to generate explosive muscle power declines

TABLE 2. Parameters measured in the SR and SSC conditions, and correlations between each parameter and eccentric strength.

\begin{tabular}{lcclcc}
\hline & \multicolumn{2}{c}{ SR Condition } & & \multicolumn{2}{c}{ SSC Condition } \\
\cline { 2 - 3 } \cline { 6 - 7 } & $0.1 \mathrm{~s}$ velocity & Peak velocity & & 0.1 s velocity & Peak velocity \\
\hline Pulling velocity $(\mathrm{m} / \mathrm{s})$ & $1.45 \pm 0.25^{*}$ & $2.32 \pm 0.24$ & & $1.73 \pm 0.32^{*}$ & $2.36 \pm 0.23$ \\
Correlations with eccentric strength & 0.10 & 0.37 & & 0.26 & $0.70^{*}$ \\
\hline
\end{tabular}

$\mathrm{SR}=$ static relaxed muscle state; $\mathrm{SSC}=$ stretch-shortening cycle.

${ }^{*} p<0.01$. 
rapidly with age. The above may suggest that the contribution of eccentric strength to muscle power output by using the SSC is not so large.

At the time of change (coupling time) in countermovement, eccentric strength might be important to catch the momentum of the counteraction by braking. This may be supported by the result that eccentric maximum strength showed a significant and high correlation with peak power in the SSC condition but not in the SR condition. However, it is important to note that eccentric maximum strength showed an insignificant correlation with initial power in which SSC potentiation was conspicuously observed.

Recently, muscular dynamics during muscle action have been clarified using ultrasonography (14). Until now, for vertical jumping (26) and walking (16), it has been understood that an agonist is stretched at the time of countermovement. However, in reality, it has been clarified that the tendon considerably contributes to the output of the lower limbs in that the tendon shortens while the muscle exerts force by isometric contraction. In this respect, Kubo et al. (25) report that athletes who have extensible tendons show high performance in jumping or sprinting. Therefore, tendon compliance may largely affect the muscle power when using SSC in the upper limbs as well. It has been reported that SSC performance is independent of maximum strength in highly trained athletes $(23,33)$. We will have to examine the relationships between tendon compliance and SSC performance in the future.

In conclusion, it was suggested that eccentric maximum strength is related to peak power using the SSC, but the contribution of eccentric maximum strength to the initial power in which SSC potentiation was conspicuously observed may be low.

\section{Practical Applications}

Because eccentric muscle training is effective for increasing maximum muscular strength $(10,12,27)$, it has been used in sports training and clinical rehabilitation. Furthermore, until now it has been suggested that eccentric maximum strength has specific exertion properties related to specific sporting events and contributes to the performance (1). However, the eccentric maximum strength of the elbow flexor muscle (biceps brachii) showed an insignificant correlation with SSC potentiation (initial power) in this study. Consequently, it was suggested that muscle power output using SSC (SSC performance) is not necessarily improved, even if maximum muscular strength is reinforced by eccentric muscle training in the upper limbs.

Miyaguchi and Demura (29) have reported that the 1RM bench press contributes to the rate of force development (initial power). Therefore, traditional weight training may be effective in improving SSC performance. Additionally, to improve SSC performance, it has been reported that first increasing the maximum muscle strength, and then performing plyometrics training such as jump training or medicine ball training, is most effective (19). In the future, we will have to examine the effectiveness of plyometrics for enhancing tendon compliance in improving SSC performance.

\section{REFERENCES}

1. Alexander, MJ. The relationship between muscle strength and sprint kinematics in elite sprinters. Can J Sport Sci 14: 148-157, 1989.

2. Amundsen, L. Muscle Strength Testing: Instrumented and NonInstrumented System. New York: Churchill Livingstone, 1990. pp. 69-112.

3. Asmussen, $\mathrm{E}$ and Bonde, PF. Storage of elastic energy in skeletal muscles in man. Acta Physiol Scand 91: 385-392, 1974.

4. Aura, $\mathrm{O}$ and Komi, PV. The mechanical efficiency of locomotion in men and women with special emphasis on stretch-shortening cycle exercises. Eur J Appl Physiol 55: 37-43, 1986.

5. Bohannon, RW. Make tests and break tests of elbow flexor muscle strength. Phys Ther 68: 193-194, 1988.

6. Bosco, C and Komi, PV. Potentiation of the mechanical behavior of the human skeletal muscle through prestretching. Acta Physiol Scand 106: 467-472, 1979

7. Cavagna, GA, Saibene, FP, and Margaria, R. Effect of negative work on the amount of positive work performed by an isolated muscle. J Appl Physiol 20: 157-158, 1965.

8. Cleak, MJ and Eston, RG. Delayed onset muscle soreness: mechanisms and management. J Sports Sci 10: 325-341, 1992.

9. Colliander, EB and Tesch, PA. Bilateral eccentric and concentric torque of quadriceps and hamstring muscles in females and males. Eur J Appl Physiol Occup Physiol 59: 227-232, 1989.

10. Colliander, EB and Tesch, PA. Effects of eccentric and concentric muscle actions in resistance training. Acta Physiol Scand 140: 31-39, 1990.

11. Daniels, L and Worthingham, C. Muscle Testing: Techniques of Muscle Examination (5th ed.). Philadelphia: WB Saunders Co., 1986. pp. 118-121.

12. Dudley, GA, Tesch, PA, Miller, BJ, and Buchanan, P. Importance of eccentric actions in performance adaptations to resistance training. Aviat Space Environ Med 62: 543-550, 1991.

13. Fukashiro, S. Biomechanical assessment of physical therapy: behavior of muscle-tendon complex during human movement. $J J p n$ Phys Ther Assoc 24: 158-163, 1997.

14. Fukashiro, S, Itoh, M, Ichinise, Y, Kawakami, Y, and Fukunaga, T. Ultrasonography gives directly but noninvasively elastic characteristic of human tendon in vivo. Eur J Appl Physiol Occup Physiol 71: 555-557, 1995.

15. Fukunaga, T. Science Dictionary of Muscle Tokyo: Asakura Publishing Co., Ltd., 2002. pp. 7-36.

16. Fukunaga, T, Ito, M, Ichinose, Y, Kuno, S, Kawakami, Y, and Fukashiro, S. Tendinous movement of a human muscle during voluntary contractions determined by real-time ultrasonography. J Appl Physiol 81: 1430-1433, 1996.

17. Furuya, K, Funato, K, Takatoh, S, Mutoh, Y, and Miyashita, M. A new isokinetic dynamometer for measuring human leg extension power. Jpn J Sports Sci 5: 669-675, 1986.

18. Griffin, JW, Tooms, RE, Vander Zwaag, R, Bertorini, TE, and O'Toole, ML. Eccentric muscle performance of elbow and knee muscle groups in untrained men and women. Med Sci Sports Exerc 25: 936-944, 1993.

19. Hakkinen, K, Komi, PV, and Alen, M. Effect of explosive type strength training on isometric force and relaxation-time, electromyographic and muscle fiber characteristics of leg extensor muscles. Acta Physiol Scand 125: 587-600, 1985.

1740 Journal of Strength and Conditioning Research 
20. Ikemoto, Y, Demura, S, Yamaji, S, Nakada, M, Kitabayashi, T, and Nagasawa, Y. The characteristics of simple muscle power by gripping-gender differences and reliability of parameters using various loads. J Sports Med Phys Fitness 46: 62-70, 2006.

21. Kellis, E and Baltzopoulous, V. Isokinetic eccentric exercise. Sports Med 19: 202-222, 1995.

22. Komi, PV. Physiological and biomechanical correlates of muscle function: effects of muscle structure and stretch-shortening cycle on force and speed. Exerc Sports Sci Rev 12: 81-121, 1984.

23. Komi, PV. Stretch-shortening cycle. In: Strength and Power in Sport P.V. Komi, ed. Oxford: Blackwell Scientific Publications, 1992. pp. 169-179.

24. Komi, PV and Bosco, C. Utilization of stored elastic energy in leg extensor muscles by men and women. Med Sci Sports 10: 261-265, 1978.

25. Kubo, K, Kanehisa, H, Kawakami, Y, and Fukunaga, T. Elasticity of tendon structures of the lower limbs in sprinters. Acta Physiol Scand 168: 327-335, 2000 .

26. Kurokawa, S, Fukunaga, T, and Fukashiro, S. Interaction between fascicles and tendinous structures during counter movement jumping investigated in vivo. J Appl Physiol 95: 2306-2314, 2003.

27. Lacerte, ML, DeLaterur, BJ, Alquist, AD, and Questad, KA. Concentric versus combined concentric-eccentric isokinetic training programs: effect on peak torque of human quadriceps femoris muscle. Arch Phys Med Rehabil 73: 1059-1062, 1992.

28. McCartney, N, Heigenhauser, GJ, Sargeant, AJ, and Jones, NL. A constant-velocity cycle ergometer for the study of dynamic muscle function. J Appl Physiol 55: 212-217, 1983.

29. Miyaguchi, K and Demura, S. Muscle power output properties using the stretch-shortening cycle of the upper limb and their relationships with a one-repetition maximum bench press. J Physiol Anthropol 25: 239-245, 2006
30. Norman, RW and Komi, PV. Electromechanical delay in skeletal muscle under normal movement conditions. Acta Physiol Scand 106: 241-248, 1979.

31. Oldfield, RC. The assessment and analysis of handedness: the Edinburgh inventory. Neuropsychologia 9: 97-113, 1971.

32. Poulin, MJ, Vandervoort, AA, Paterson, DH, Kramer, JF, and Cunningham, DA. Eccentric and concentric torques of knee and elbow extension in young and older men. Can J Sports Sci 17: 3-7, 1992.

33. Schmidtbleicher, D. Strength training: part 1. Classfication of methods. Sci Period Res Technol Sport W-4: 1-12, 1985.

34. Stauber, WT. Eccentric action of muscles: physiology, injury, and adaptation. Exerc Sport Sci Rev 17: 157-185, 1989.

35. Takamatsu, K, Aida, $\mathrm{H}$, and Zushi, K. Effects of isometric and eccentric preliminary muscle contraction on elbow flexion velocity: with special reference to load and range of motion during concentric contraction. Jpn J Phys Educ Health Sport Sci 36: 127-139, 1991.

36. Tauchi, K, Yoon, S, and Takamatsu, K. The difference between the upper limbs and the lower limbs in individuals on force output characteristics during stretch-shortening cycle movement. Jpn J Phys Educ Health Sport Sci 47: 533-546, 2002.

37. Walshe, AD, Wilson, GJ, and Ettema, GJC. Stretch-shorten cycle compared with isometric preload: contributions to enhanced muscular performance. J Appl Physiol 84: 97-106, 1998.

38. Walshe, AD, Wilson, GJ, and Murphy, AJ. The validity and reliability of a test of lower body musculotendinous stiffness. Eur J Appl Physiol 73: 332-339, 1996.

39. Wilson, GJ, Murphy, AJ, and Pryor, JF. Musculotendinous stiffness: its relationship to eccentric, isometric, and concentric performance. J Appl Physiol 76: 2714-2719, 1994.

40. Yamamoto, T. Measurement and Assessment for Practical and Scientific Conditioning [in Japanese]. Tokyo: Book House HD, 2001. pp. 22-32. 